\title{
Asymmetric Movements between the US and Hong-Kong Stock Market Price Indices
}

\section{Francisca Mendonça Souza', Luciane Flores Jacobi", Roselaine Ruviaro Zanini"', Adriano Mendonça Souza Iv}

\begin{abstract}
In the financial field, we are often faced with data that are somewhat non-linear. Thus, this research investigates the asymmetric behavior of the series through the Threshold Autoregressive and Momentum Threshold asymmetry tests between price index using diary data between of the total price index of actions in stock markets of United States of America (S\&P500) and Hong Kong (HANG SENG), from January 2, 1995 to March 29, 2013. With the empirical analysis it was possible to see the asymmetry in the series analysed through the TAR and M-TAR models. These oscillations demonstrate that behaviors are distinct in contractions and expansions.
\end{abstract}

Keywords: TAR and M-Tar, CUSUM, Asymmetric test, Price index, Interst rates 


\section{INTRODUCTION}

Financial phenomena are characterized by oscillatory movements, which occur in response to changes in the explanatory variables. The stock market is typically characterized by joint movements in price indexes or listed securities which, in turn, rely on exogenous and endogenous financial and economic variables. These movements (upwards or downwards) occur in response to changes in the explanatory variables. However, the extent of these responses may be different for positive and negative changes of the same magnitude, in which case we say that the variables exhibit asymmetric adjustment over the business cycle (Menezes et. al. 2006), i.e. positive and negative shocks of the same magnitude have effects of different magnitude for each variable.

While there are strong theoretical signals that the stochastic residuals of many financial models should not be exactly symmetric around their mean values, it has been more difficult to showed empirical proofs of such asymmetry, mostly because it may be confounded with other types of data nonlinearities. In this study we analyze whether price index movement asymmetry occurs between the United States and Hong Kong.

The asymmetry of comovements between economic and financial variables is a special case of a broader class of models commonly referred to as nonlinear models. For example, Granger and Teräsvirta (1993) study in depth the modeling of nonlinear economic relationships. Examples of such relations in economics include the attitude of investors towards risk and the process of generation of financial data, such as equity returns, dividends, interest rates and so on. Moreover, the performance of an economy also presents strong signs of nonlinear behavior such as, for example, business cycles, production functions, growth rates, unemployment, among others.

There exist in the literature a large number of models proposed for measuring asymmetrical relationships between variables (see, e.g. Tong, 1983, 1990). In our context, we are interested in analyzing a particular class of models known as 
Threshold AutoRegressive (TAR - or switching regime) and Momentum Threshold Auto Regressive (M-TAR) models to ascertain the extent of asymmetry in the relationship between stock markets. In the context of nonstationarity, asymmetric cointegration tests were developed by Enders and Siklos (2001) using a modified error correction model based on the original EG test procedure (see, Gjerde and Saettem, 1999; Rapach, 2001).

\section{METHODOLOGY}

The long-term equilibrium coefficient between two time series $x_{1 t}$ and $x_{2 t}$ can be estimated using a standard regression model of the type identified in the expression

$$
x_{1 t}=c_{0}+\beta x_{2 t}+v_{t}
$$

where $v_{t}$ is a random disturbance which can be autocorrelated. The parameter $\beta$ indicates the magnitude of the adjustment of the variations in $x_{1 t}$ and $x_{2 t}$ and measures the elasticity of the long-run term between the two variables, when they are measured in logarithms. If $\beta<1$ the changes in $x_{2 t}$ are not completely transmitted to $x_{1 t}$ and so forth. Since the main interest focuses on the asymmetric behavior of the residual variable $v_{t}$, in the second step we obtain the estimated OLS $\rho$ of the following dynamic model:

$$
\Delta v_{t}=\rho v_{t-1}+\varepsilon_{t}
$$

where $\varepsilon_{t}$ is a random white noise disturbance and the residuals of equation (1) are used to estimate equation (2).

The convergence of the model is achieved if $-2<\rho<0$ meaning that, in this case, equation (1) is an attractor such that $v_{t}$ can be written as an error correction model (Enders and Siklos 2001 ).

Equation (2) can, alternatively, be estimated in such a way as to allow taking into account different adjustment processes for positive and negative variations as follows 


$$
\Delta v_{t}=I_{t} \rho_{1} v_{t-1}+\left(1-I_{t}\right) \rho_{2} v_{t-1}+\varepsilon_{t}
$$

where $\rho_{1}$ and $\rho_{2}$ represent the effects on the variation of $v_{t}$ caused by positive and negative variations in the system. It is the Heaviside indicator function, such that

$$
I_{t}= \begin{cases}1 & \text { se } \xi_{t-1} \geq \tau \\ 0 & \text { se } \xi_{t-1}<\tau\end{cases}
$$

Working in equation (4) $\xi_{t-1}=\boldsymbol{v}_{t-1}$, then the model (3) is called TAR. This model takes into account the existence of mechanisms to correct the error for different positive and negative values $v_{t}$. A sufficient condition for the stationarity of $v_{t}$ is $-2<\left(\rho_{1}, \rho_{2}\right)<0$. If $\rho_{1}=\rho_{2}$ then we have a particular case of (3) in which the adjustment is symmetric and equation (3) reduces to equation (2). Note that, given the need to ensure that a process $\varepsilon_{t}$ is white noise, the expressions (2) and (3) can also contain offset values $\Delta v_{t}$, and the optimal number of lags determined by a suitable criteria information. When $\boldsymbol{v}_{t-1}$ is above its equilibrium value, in the long term, the adjustment is given by $\rho_{1} v_{t-1}$; conversely, when $v_{t-1}$ is below its equilibrium value, in the long term, the adjustment is given by $\rho_{2} v_{t-1}$. If in equation (4), $\xi_{t-1}=\Delta v_{t}$ then the model (3) is called MTAR. This model takes into account the fact that the variation of $v_{t}$ depends on the change that occurred in the previous period $v_{t-1}$. The other conditions of the TAR model also apply to the case of the M-TAR model. Note that the TAR and M-TAR models are special cases of a broader class of nonlinear models described in literature by Switching Models in which the threshold of regime change and the number of lags can be determined in the process or how the endogenous or exogenous SETAR and STAR as TAR and M-TAR model. As our interest is to analyze the different behavior for positive and negative shocks in the system, the TAR and M-TAR models are suitable.

The TAR model is designed to capture asymmetrically deep movements in the series of deviations from the long run equilibrium, while the M-TAR is useful to 
capture any abrupt movements asymmetrically in this series (Enders and Granger, 1998). If, for example, if the TAR model $-1<\rho_{1}<\rho_{2}<0$, then the negative phase $\boldsymbol{v}_{t}$ tend to be more persistent than the positive phase. On the other hand, if the model M-TAR for example, we have $\left|\rho_{1}\right|<\left|\rho_{2}\right|$, then, the model exhibits a small decline for $\Delta v_{t-1}$ positive but a substantial decrease in $\Delta v_{t-1}$ negative. This means that increases tend to persist over time, but quickly decreases tend to revert to the attractor.

In this context, it can make a set of statistical tests on the coefficient estimates and on the model of the residual, in order to confirm the validity of the correction error set (3) model and thus if the adjustment is symmetrical or not. The relevant tests on the estimated coefficients are: (1) $\mathrm{H}_{0}$ : $\max \left(\rho_{1}, \rho_{2}\right)=0$, for which, you get the sample values $t$ of the statistic and, (2) $\mathrm{H}_{0}: \rho 1=\rho 2=0$, for which the sample values $F$ statistical. The restriction that the adjustment is symmetric $\left(\rho_{1}=\rho_{2}\right)$ can also be tested based on the usual $F$ statistic.

If the variables in the long run equation are not stationary, the critical values of $t$ and $F$ distributions are not valid to access the level of significance (1) and (2). In this case, the asymptotic distribution of the critical values reported by Enders and Granger (1998) and Enders and Siklos (2001) can be used to determine if the null hypothesis cannot be rejected cointegration (tests (1) and (2)). If it is rejected, then it is possible to test the existence of asymmetric adjustment using the critical values of the $F$ distribution, as $\rho_{1}$ and $\rho_{2}$ converge in this case, for a multivariate normal distribution (Enders and Granger, 1998). It should be noted that the regime transitions suffered by the series have different characteristics, i.e. they can be abrupt and evaluated by the TAR model initially proposed by Tong (1978).

The methodological steps for the analysis of the asymmetric behavior used in this study are described below.

1) First, the data are tested in relation to instability, stationarity and structural breaks aiming to verify the presence of unit roots through CUSUM and the test Lanne et al. (2002).

2) After testing for unit roots, we analyze the behavior of the variables under 
study in terms of the asymmetry TAR and M-TAR tests.

For this purpose were collected from the base of DataStream data, daily observations of the total price index of actions in stock markets of United States of America (S\&P500) and Hong Kong (HANG SENG), from January 2, 1995 to March 29, 2013, totaling 4761 observations and consider in its formulation only five days of operation per week. The series collected consider five days a week, with the daily stock market index series having been converted to base 100, and the yields are then calculated on the basis of the first differences of the logarithmic price series. The series collected consider five days a week, with the daily stock market index series having been converted to base 100, and the yields are then calculated on the basis of the first differences of the logarithmic price series. The choice of the markets identified above was due to the fact that belongs to the group of economies that may achieve greater participation and importance in the international scenario, with diversified characteristics. The United States has the world's largest and most influential financial market and Hong Kong is the second largest stock exchange in Asia and it's among the top 10 in the world. The diversity that characterizes these markets is expected to make it possible to verify the extent to which the respective stock market indices are influenced by the time structure of interest rates with the positive and negative impacts generating distinct effects.

\section{RESULTS AND DISCUSSION}

Verification of the structural break is crucial to separate the number of other types of deviations such as unit roots, ie, the non-stationary, since the structural break has potentially similar effect on the properties of second order of a time series. For this investigation will be used the CUSUM test based on a CUSUMQ of the recursive residual test. The null hypothesis is that the coefficient vector $\beta$ is the same for the entire period and the alternative hypothesis is that there is a disturbance in the variance. It is a general test and does not require a priori specification, when a 
structural break occurs. The rejection rule test that will be discussed below is that rejects the null hypothesis of no structural break whenever exceeds the critical value obtained by the supreme standard Brownian bridge.

$$
U_{k^{*}}=\frac{1}{\sqrt{\hat{w}}} \max _{1 \leq k \leq T}\left|D_{K}\right|
$$

The CUSUM test is based on the cumulative sum of the recursive residuals. The decision rule is that the null hypothesis of absence of structural failure is rejected whenever (5) exceeds the critical value obtained. This option plots the cumulative sum together with the $5 \%$ critical lines. The test find's parameter instability if the cumulative sum goes outside the area between the two critical lines. Tests for stability and CUSUMQ CUSUM procedure can be analysed in the following Figure 1.

Figure 1 - Stability test to price index in the period from 01/02/1995 to 03/29/2013: figure (a) CUSUM United States (S\&P500), figure (b) CUSUMQ United States (S\&P500), figure (c) CUSUM Hong Kong (HANG SENG), figure (d) CUSUMQ Hong Kong (HANG SENG) 


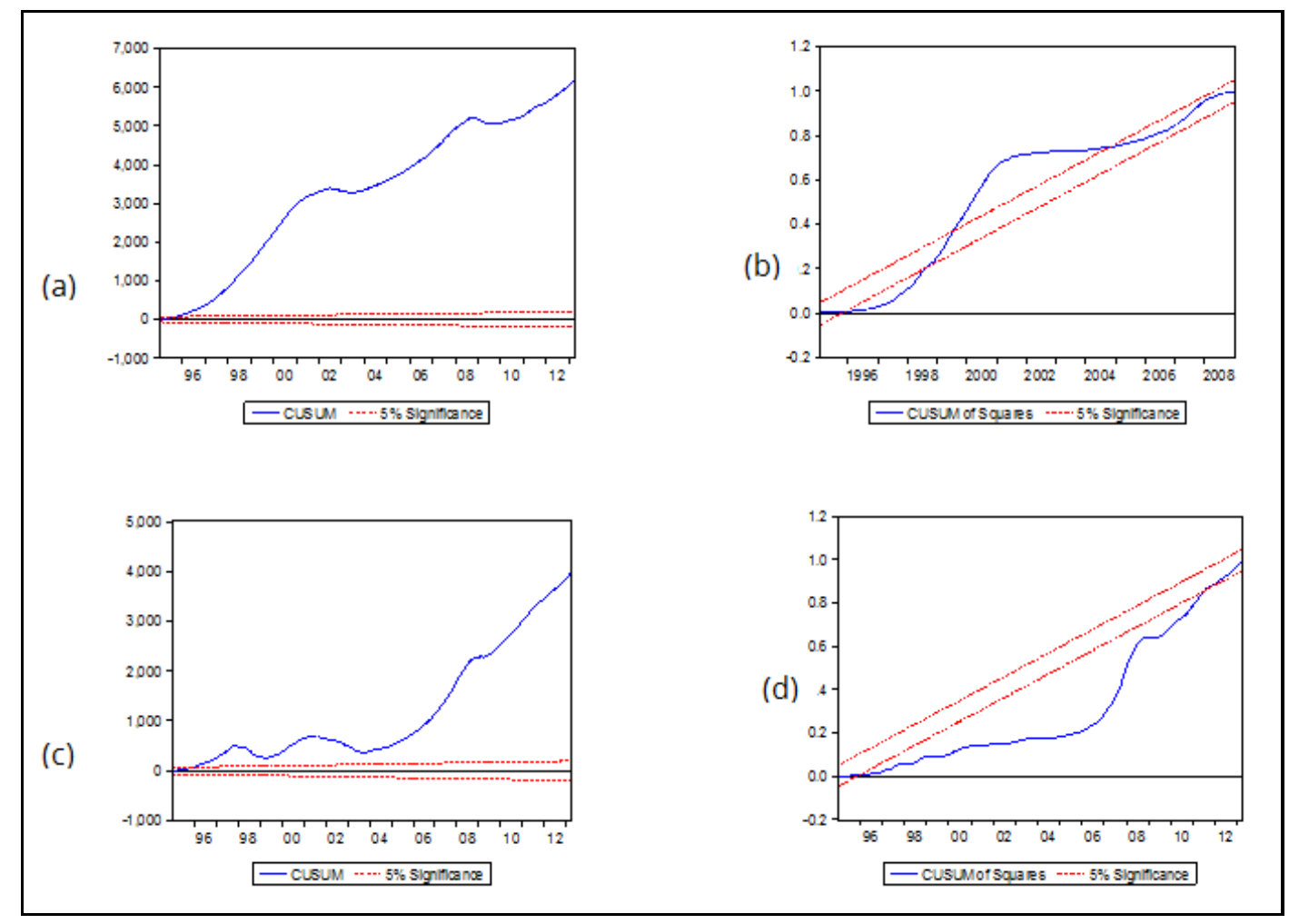

Analyzing the above figures, the results of the test CUSUMQ agree with the CUSUM test. Shows large instability of the parameters, which is visible in tests, the CUSUM and the CUSUMQ test. The stability results of CUSUM tests and CUSUMQ denote the existence of structural breaks in time series employed. In the case of a structural break in the deterministic trend, the conventional unit root tests such as Augmented Dickey-Fuller (ADF), lose their power and induce unreliable conclusions. Then, for testing stationary series and possible dates structural breaks we use the test by Lanne et al. $(2001,2002)$.

To test the stationarity series we used the Lanne et al. (2001, 2002), where the null hypothesis that the variable has a unit root - I(1) was not rejected, as shown in Table 1. The non-stationary series in level become stationary after the first difference.

Table 1 - Unit root tests Lanne et al. (2001, 2002), for the variable price index

\begin{tabular}{l|l|l}
\hline Variables & t-stat & Date of break \\
\hline United States (S\&P500) & $-1,316$ & $09 / 29 / 2008$ \\
\hline Hong Kong (HANG SENG) & $-2,530$ & $10 / 29 / 2008$ \\
\hline \multicolumn{2}{l}{ Source: Data compiled by the author worked (software used: JMulti). }
\end{tabular}

Note: The critical test values (Lanne et al 2002) are as follows: (i) model with constant: -3.48 (1\%) and -2.88 (5\%); (ii) model with constant and deterministic trend: -3.55 (1\%) e -3.03 (5\%).

${ }^{* *}$ ) significance $1 \%$; 
$(* *)$ and significance 5\%;

(*). Price Index, previous logarithmic.

Table 1 presents the results of the stationary test for the series at levels that indicate that all two variables under study are I(1), becoming stationary in differences. This test when applied to the RPI variable suggests a regime change in the year 2008 for the United States and Hong Kong. The date of 29/09/2008 will be set in this study as the point of regime change.

Table 2 shows that for both models, the null hypothesis of non-cointegration is rejected, because the F-values for the TAR and M-TAR models are found between 7.81 and 8.40, respectively. In addition, the results from the TAR model suggest that the null hypothesis of symmetry cannot be rejected. Through the TAR and M-TAR asymmetry tests, the behavior of the two long-term stock market indices was analysed.

Asymmetry can arise from a number of factors, e.g. on the stock market, asymmetry can occur because investors seek to minimize losses and maximize risks. Risk aversion may encourage economic agents to react quickly to unfavorable news and to react more slowly to favorable news (Menezes et al., 2006). On the other hand, asymmetries can arise from potential losses resulting from overstated markets (Siklos, 2002). This idea is corroborated by Menezes (2004, 2006) and Souza (2016). Therefore, we apply the tests proposed by Enders and Siklos (2001) to analyse the issue of TAR adjustments that allow capturing deep asymmetric movements in the long-term equilibrium deviation and M-Tar series that allow capturing abrupt asymmetric movements on the series in the context of cointegration. It was evaluated through the following hypotheses: 1) $\left.\mathrm{H}_{0}: \max \left(\rho_{1}, \rho_{2}\right)=0 ; 2\right) \mathrm{H}_{0}: \rho_{1}=\rho_{2}=0$, that the null hypothesis of non-cointegration between the included variables is rejected in the long-term model. Already the test 3) $\mathrm{H}_{0}: \rho_{1}=\rho_{2}$ allows the asymmetry to be assessed. The negative sign of the coefficients guarantees the stationarity of the variables.

Table 2 - Asymmetric test Price Index for US (S\&P500) and HK (HANG SENG) 02/01/1999 a 29/03/2013 


\begin{tabular}{l|l|l|l|l|l}
\hline Test & $\rho_{1}$ & $\rho_{2}$ & $\frac{\max \left(\rho_{1}, \rho_{2}\right)=0}{\mathrm{t}-\max }$ & $\frac{\rho_{1}=\rho_{2}=0}{\Phi}$ & $\frac{\rho_{1}=\rho_{2}}{\mathrm{~F}(\mathrm{p} \text {-value })}$ \\
\hline USA (S\&P500) / & & & & \\
HK (HANG SENG) & $-0,0019$ & $-0,0020$ & $-18,1115^{* *}$ & $113,3611 * *$ & $160,7181(0.000)$ \\
TAR & $-0,0012$ & $-0,0019$ & $-20,1450 * *$ & $119,8029 * *$ & $153,4255(0.000)$ \\
M-TAR & Notes: \\
Ho: $\max \left(\rho_{1}, \rho_{2}\right)=0 ;$ critical values: $-2.51(1 \%),-2,10(5 \%)$ in TAR e $-2.42(1 \%),-1.99(5 \%)$. \\
M-TAR. $H_{0}: \rho_{1}=\rho=0 ;$ critical values: $7.81(1 \%), 5.79(5 \%)$ in TAR $8.40(1 \%), 6.28(5 \%)$ in M-TAR. \\
Ho: $\rho_{1}=\rho_{2} ;$ critical values of distribution $F .4757$ observations after adjustments. \\
* significance $1 \%$, \\
** significance 5\%.
\end{tabular}

Stock markets have different reactions to the same news, depending on the state of the economy. Negative news can have a positive impact when the market is expanding and a negative impact during recessions. With the empirical analysis it is possible to see the asymmetry in the series analysed through the TAR and M-TAR models. These oscillations demonstrate that behaviors are distinct in contractions and expansions.

\section{CONCLUSIONS}

Several explanations have been proposed for this asymmetric effect, one of which is called volatility feedback effect, which is based on the idea that when the volatility of an asset increases, the risk is greater and the return on this asset increases. The asset is less attractive and, therefore, its demand decreases, causing its price to fall. This explanation assumes that the increase in volatility proceeds the fall in price. Another explanation is that the asymmetric effect is due to investors selling their securities when the market is falling. This behaviour leads not only to a steeper drop in the price but also to increase in volatility. The adjustment TAR e M-Tar test confirmed that positives and negative movements between US and HK, ie, are asymmetric and suggests there is existed more than regime between interest rates and price index of the markets analysed. This analyse highlight the importance of modelling the cyclical behaviour of stock markets and identify that theses stock markets has a different reaction to the same news, depending on the state of the economy. Once the asymmetry has been analysed through the TAR and M-TAR 
models, it is suggested that a future study should analyse the effect of the impact of shocks through the transition phenomenon, using the STR models, which are characterized by describing the regime change through the continuous transition function.

\section{ACKNOWLEDGEMENTS}

This study was financed in part by the Coordenação de Aperfeiçoamento de Pessoal de Nível Superior - Brasil (CAPES) - Finance Code 001. Process-BEX - 5416/108, through the UNIDE-IUL Research Center/ ISCTE-IUL.

\section{REFERENCES}

ENDERS W, GRANGER CWJ. Unit-root test and asymmetric with an example using the structure of interest rates. Journal of Business \& Economic Statistics. 1998;16, 304311.

ENDERS W, SIKLOS P. Cointegration and threshold adjustment. Journal of Business \& Economic Statistics. 2001;19(2), 166-176.

GRANGER CW], TERÄSVIRTA T. Modelling Nonlinear Economic Relationships. New York: Oxford University Press; 1993.

GJERDE, SAETTEM F. Causal relations among stock returns and macroeconomic variables in a small, open econom. Journal of International Financial Markets, Institutions and Money. 1999;9, 61-74.

LANNE M, LÜTKEPOHL H, SAIKKONEN P. Test Procedures for Unit Roots in Time Series with Level Shifts at Unknown Time. Humboldt University of Berlin Discussion Paper. 2001;(39).

LANNE M, LÜTKEPOHL H, SAIKKONEN P. Comparison of unit root tests for time series with level shifts. Journal of Time Series Analysis. 2002;23, 667-685.

MENEZES R, DIONÍSIO A, MENDES D. Mutual information: a measure of dependency for nonlinear time series. Physica A. 2004;344, 326-329.

MENEZES R, FERREIRA NB, MENDES DA. Co-movements and asymmetric volatility in the Portuguese and US stock markets. Nonlinear Dynamics. 2006; 44(1), 359-366. 
RAPACH DE. Macro shocks and real stock prices. Journal of Economics and Business. 2001;53, 5-26.

SOUZA FM. Efeitos de Contágio das Taxas de Juro a Longo Prazo na Rendibilidade dos Índices Bolsistas Internacionais: Um Modelo com Quebras Estruturais, Persistência e Heterocedasticidade Condicionada [thesis]. Lisboa: Instituto Universitário de Lisboa; 2016. 147 p.

SIKLOS PL. Asymmetric adjustment from structural booms and slumps. Economic Letters. 2002;77, 329-333.

TONG H. On a threshold model. In: Chen CH. (Ed.), Pattern Recognition and Signal Processing. Sijthoff and Noordhoff, Amsterdam; 1978.

TONG H. Threshold Models in Non-Linear Time Series Analysis. New York: SpringerVerlag; 1993.

TONG H. Non-Linear Time-Series: A Dynamical Systems Approach. Oxford: Oxford University Press; 1990. 